\title{
A MORPHOLOGICAL DOMINANT POINTS DETECTION AND ITS CELLULAR IMPLEMENTATION
}

\author{
J.Richefeu, A.Manzanera \\ École Nat. Sup. de Techniques Avancées \\ Unité d'Électronique et d'Informatique \\ 32, Boulevard Victor \\ 75015 Paris, France
}

\begin{abstract}
This paper addresses the problem of detection of dominant points also called interest points or key points in the literature. We present a new algorithm to compute dominant points on a gray level image. It is founded on mathematical morphology, and it is adapted to SIMD cellular, level sets based, Boolean computation. It is also compliant with the morphological scale-space framework. Cellular implementation and results are presented.
\end{abstract}

Key words: dominant point, mathematical morphology, level set, skeleton, parallel cellular machine

\section{INTRODUCTION AND PRELIMINARIES}

An interest point is a location in the image where the signal changes in several directions, thus carrying more information. Examples include corners (L-points with more or less important angle) and T-junctions, as well as locations where texture varies significantly [13]. Such points are useful in many applications, such as: image matching (correlation) as part of motion analysis or image registration processes and $3 \mathrm{D}$ reconstruction in computer vision or image synthesis.

A very large set of interest point detectors has already been proposed in the literature. References for quasi-exhaustive surveys are [4][13]. We can file it into three categories.

Approaches in the first group involve first extracting edges as a chain code and then searching for points of maximal curvature [1][4], or performing a polygonal approximation on the chains and then searching for the line segment intersections [7].

The second group consists of approaches working directly on the gray-level image. These techniques are based either on empirical local operators [12] or on the measurement of the gradients and curvatures of the surface. A great deal of effort has been devoted to this second type of approach in order to detect corners and vertices [5].
The last group deals with theoretical models of the signal in order to obtain sub-pixel precision. Such methods are used only for specific types of interest points like corners [6].

Few authors have considered morphological dominant points. One work [15] deals with binary images to detect convex corner points on closed contours. It is based on residues of morphological opening.

Our approach consists in defining an interest function based on a measure of local curvature on the level sets of the gray-level image (section 2). We then use the skeleton to approximate the computation of the high curvature points (section 3).

This algorithm is well suited to SIMD cellular processing. We present the implementation on the programmable retina (section 4) that allows efficient parallel level sets based image processing. Results are finally discussed (section 5).

\section{THE INTEREST FUNCTION}

In this section, we present the concept of morphological level set processing used for our dominant points detection. Then we use it to define a gray-level interest function from the binary local curvature.

\section{1. level set processing}

Mathematical morphology is a nonlinear image processing framework developed by G.Matheron and J.Serra [14]. Level sets represent a natural way to extend the morphological operator from binary to gray-level images.

Let $E$ be any set. A binary image $X$ is a subset of $E$. Let $K$ be an interval $[0, n]$ of $\mathbb{N}$. A grayscale image is a function $I$ from $E$ to $K$.

Such a grayscale image $I$ can be represented by its level sets $\left\{I_{t}\right\}_{t \in[1, n]}$ defined as follows:

$$
I_{t}=\{p \in E \mid I(p) \geq t\}
$$


So reciprocally,

$$
I(p)=\max \left\{t ; p \in I_{t}\right\}
$$

\section{2. local curvature and definition of interest function}

For binary images, one natural interest function is provided by the local curvature.

In Euclidian space, the curvature is defined as the instantaneous rate of slope change [1]. Assume that $y(x)$ represents a curve function, and $R_{p}$ is the curvature at point $p$, then $R_{p}$ is represented by its derivatives :

$$
R_{p}=\frac{\frac{\partial^{2} y}{\partial x^{2}}}{\left[1+\left(\frac{\partial y}{\partial x}\right)^{2}\right]^{3 / 2}}
$$

The curvature is reciprocal of the radius of the maximal inscribed circle tangent to the curve at point $p$.

Let $\stackrel{\circ}{X}$ be the contour of $X$. The interest function $\varphi_{X}$ associated with the binary image $X$ is then defined as:

$$
\varphi_{X}(p)=\left\{\begin{array}{l}
R_{p} \text { if } p \in \stackrel{\circ}{X} \\
0 \text { elsewhere }
\end{array}\right.
$$

According to the level sets framework, we can now define the interest function $\phi$ for the gray-level image $I$ by the sum of the binary interest functions $\varphi_{I_{t}}$ over the level sets $I_{t}$ of the image.

$$
\begin{aligned}
\phi: E & \rightarrow K \\
p & \mapsto \sum_{t=1}^{n} \varphi_{I_{t}}(p)
\end{aligned}
$$

It can be observed that by replacing in equation (4) $R_{p}$ by a constant value we obtain the morphological gradient. So the function $\phi$ provides a measure of interest on gray-level images by weighting the local contrast by the curvature.

\section{THE SKELETON}

As the interest points will be computed by thresholding the interest function, it can be sufficient to compute only the high curvature points. Such points can be found by using a thinning algorithm.

The skeleton was introduced by H.Blum [3] as the medial axis which is the union of the centers of the maximal inscribed balls. According to H.Blum, the highest curvature points are located on the maximal inscribed circles whose centers are the extremities of branches of the skeleton. Furthermore, this curvature is reciprocal of the distance between the contour and the extremal point of the skeleton.

For discrete images, one popular approach to object skeletonizing is to thin an object (i.e. remove iteratively contour points while preserving topology and geometry) until a minimally connected set of pixels is achieved.

The skeleton algorithm used in our dominant point detector is the MB1-Hybrid thinning algorithm detailed in [11]. The advantages of this algorithm are: (1) it has a certain rotation invariance; (2) it produces few spurious branches; (3) its computational cost is low; (4) there exist 8-connected and 4-connected versions having the same geometrical properties.

One complete iteration of the MB1-Hybrid thinning algorithm actually corresponds to one iteration of a fully-parallel algorithm followed by four iterations of a semi-parallel directional algorithm in the four directions successively

(North, South, East and West). Such a combination allows better rotation invariance. Approximately, it produces a skeleton branch for every angle inferior to $\pi / 2$ in any orientation. Boolean templates of the algorithm and implementation details can be found in [11].

In order to obtain the positive high curvature points on a binary image, we compute one complete iteration of the MB1-Hybrid skeleton then we calculate the extremal points of the skeleton (those which have one neighbor only in the image). In the same way, the negative high curvature points are computed thanks to the exoskeleton (skeleton of the complementary of the image). Figure 2 shows an example of this process.
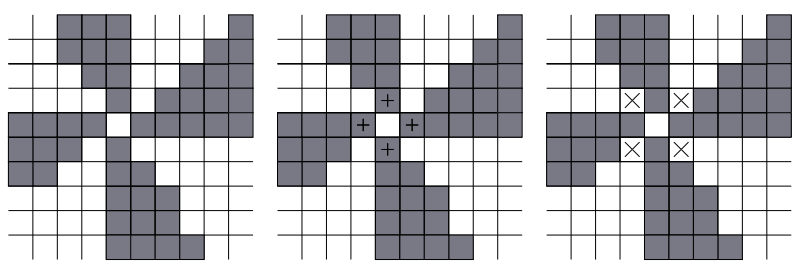

Figure 1: Duality junction/corner and the discrete Jordan's theorem.

The previous procedure allows us to compute the convex and concave corner points (L-points). We can also calculate the junction points (T-points) by finding the multiple points of the skeletons. Nevertheless, by duality, the junction points are associated to corner points of the complementary, and the multiple points of the skeleton (resp. exoskeleton) will be detected by the presence of extremal points of the exoskeleton (resp. skeleton), as long as different connexity levels are used for the image and its complementary (discrete Jordan's theorem in the square grid [9]). For example, on the configuration shown on Figure 1, the junction is detected by either the presence of 4 extremal points of the 4-connected exoskeleton (points marked "+") or by the presence of 4 extremal points of the 4-connected skeleton (points marked "x").

This procedure will be performed on every level set of the gray-level image in order to obtain the interest function 

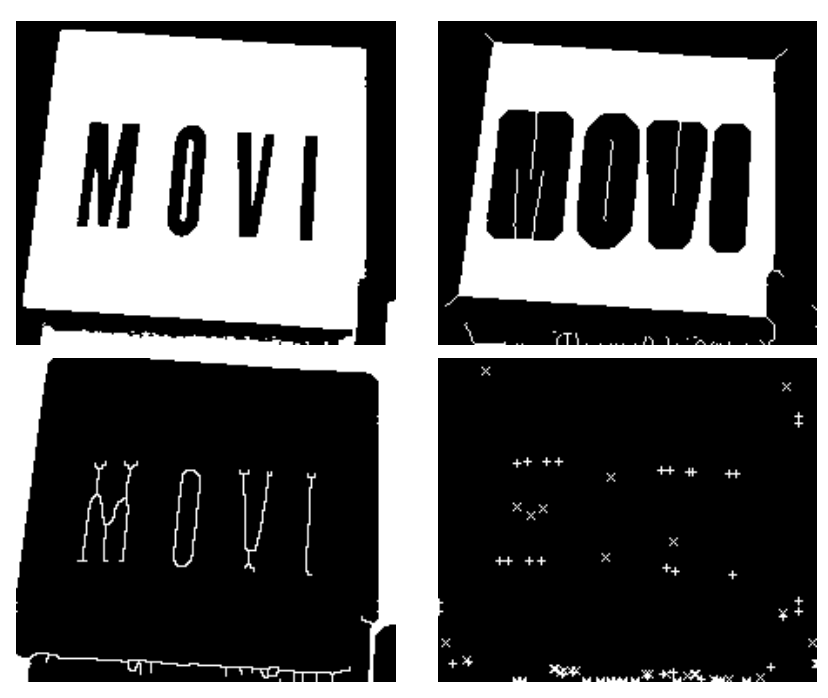

Figure 2: The original binary image, 8-connected skeleton (5 iterations), 4-connected exoskeleton (5 iterations) and union of the extremal points of the skeleton (points marked " $\mathrm{x}$ ") and the exoskeleton (points marked "+").

by summing the results.

\section{IMPLEMENTATION}

The programmable retina [2] is a CMOS array sensor in which a Boolean elementary processor has been integrated inside every pixel, so it is a cellular SIMD machine with optical input. This architecture has been shown [10] to be well suited for non-linear (Boolean) and level-by-level processing, thanks to an analog-to-digital conversion by multiple thresholding, that allows data processing during the acquisition.

In such architecture, the procedure used to detect dominant points is the following one :

For every time index $t$ :

1. acquisition of level set $I_{t}$ by threshold;

2. copy and inversion of $I_{t}$ and $I_{t}^{c}=\operatorname{NOT}\left(I_{t}\right)$;

3. one iteration of thinning of images $S\left(I_{t}\right)$ (skeleton) and $S\left(I_{t}^{c}\right)$ (exoskeleton);

4. search for extremal point ep $(S(I))$ and ep $\left(S\left(I_{t}^{c}\right)\right)$;

5. logical "OR" between the two images $F_{t}=\operatorname{ep}\left(S\left(I_{t}\right)\right)$ OR $\operatorname{ep}\left(S\left(I_{t}^{c}\right)\right)$;

6. accumulation of the sum $F=F+F_{t}$

endFor
The result of the previous procedure $F$ is the interest function, dominant points are then obtained by thresholding $F$.

The overall number of elementary Boolean parallel operations performed by level $t$ is less than 150 . So if the programmable retina is controlled at a $10 \mathrm{Mhz}$ frequence, the computation time for all the level sets is less than $4 \mathrm{~ms}$ for 256 gray-levels and less than $0.5 \mathrm{~ms}$ for 32 gray-levels.

\section{RESULTS}

We show in this section some results obtained with our simulation software of the retina architecture. The first test image is taken from the "MOVI" motion of the INRIA database and the second is a detail of the "Goldhill" test image.

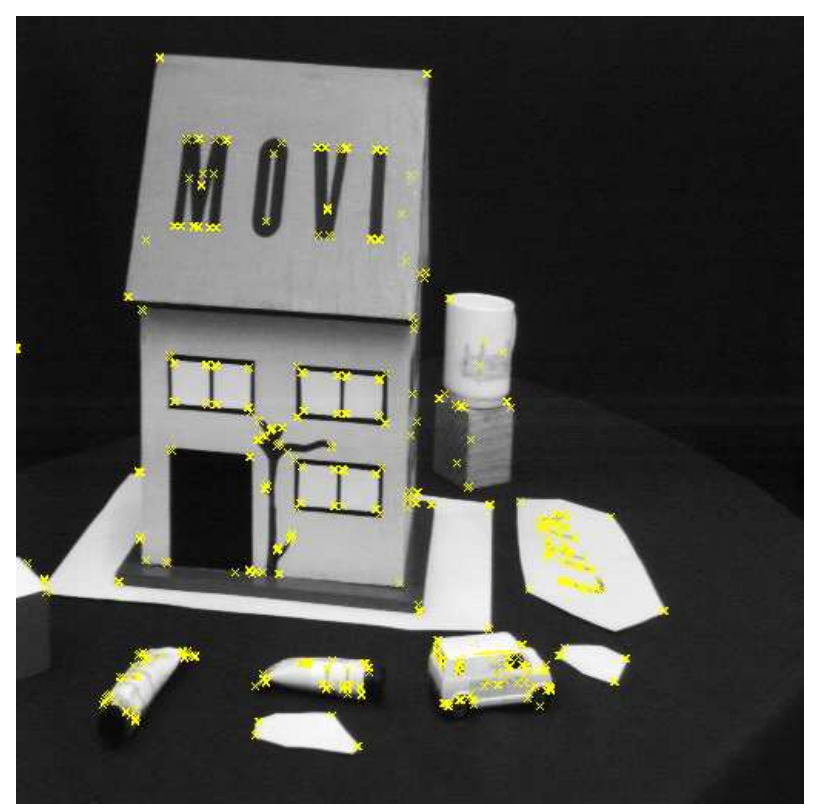

Figure 3: The resulting dominant points detection image on "MOVI" test image.

Figure 3 shows an example of dominant points detection computed on 256 gray-levels by thresholding the interest function at the value of 13 . See Figure 4 for a detail.

A hierarchical selection of the level sets defines a morphological scale-space [8]. As our interest function is a sum over the level sets, it is necessarily decreasing as the morphological scale increases. Our detector is thus compliant with such morphological scale-space as no new dominant point appears as the scale increases. An example is shown on Figure 5 using the alternated sequential filters by reconstruction. 

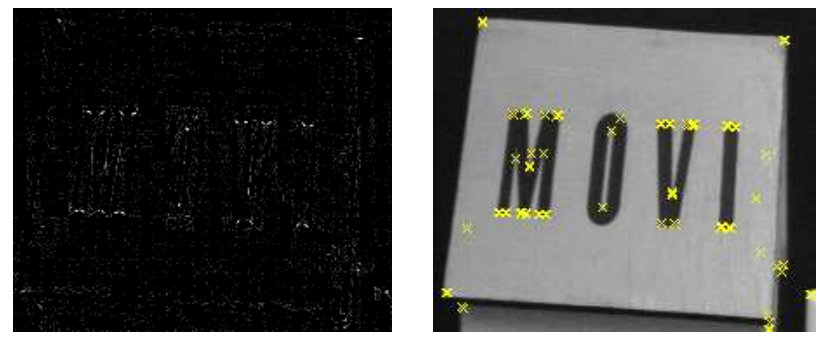

Figure 4: A detail of the interest function computed on 256 gray levels and the corresponding dominant points for the threshold at the value of 13 .

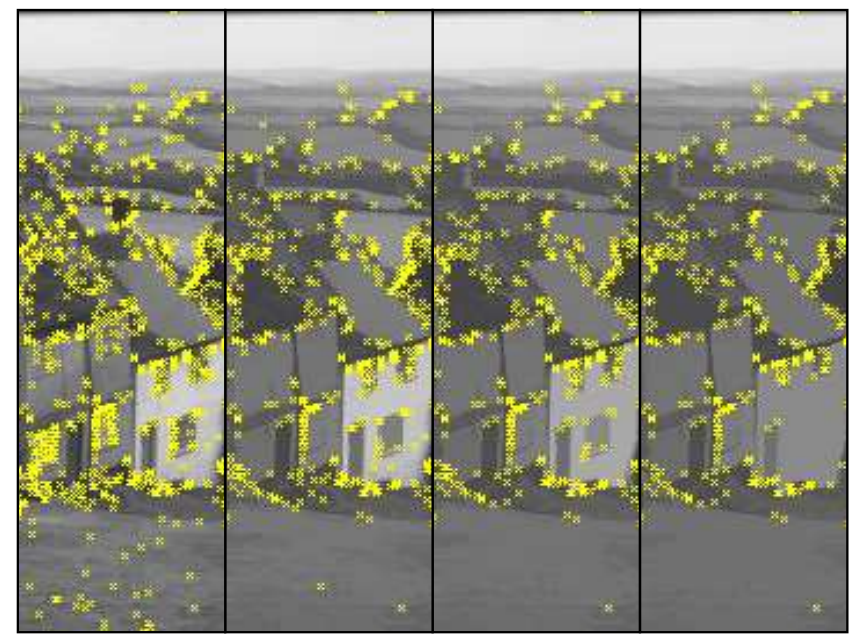

Figure 5: Dominant point detection over a morphological scale-space on a detail of the "goldhill" image

\section{CONCLUSION}

In this paper, we have proposed a new dominant point detection algorithm based on mathematical morphology and in particular on skeletons. The algorithm can be executed in parallel cellular machines like the programmable retina very efficiently. The MB1-Hybrid thinning algorithm used in the implementation is a good trade-off between rotation invariance, efficiency and robustness. The number of dominant points can be tuned according to the contrast thanks to thresholding and according to the size of the level sets through the scale-space framework.

This study is part of our current research in the field of motion analysis. In fact, dominant points detection is the first step involved in correlation to match different images on a sequence. In following works, we will automatically detect and study motion on a video system based on the artificial retina.

\section{REFERENCES}

[1] H. Asada and M. Brady, "The curvature primal sketch", IEEE Trans. PAMI, Vol. 8(1), pp. 2-14, 1986.

[2] T.M. Bernard, "Des rétines artificielles intelligentes", phD Thesis, Université Paris-Sud, 1992.

[3] H. Blum, "A transformation for extracting new descriptors of shape", Symp. Models for Perception of Speech and Visual Form, Weiant Whaten-Dunn Ed., MIT Press, Cambridge, MA, 1967.

[4] R. Deriche and G. Giraudon, "A computional approach for corner and vertex detection", Int. Journal of Computer Vision, vol. 10(2), pp. 101-124, 1993.

[5] C. Harris and M. Stephens, "A combined corner and edge detector", Alvey Vision Conference, pp. 147-151, 1988.

[6] F. Heitger et al., "Simulation of neural contour mechanism: from simple to end-stopped cells", Vision Research, vol. 32(5), pp. 963-981, 1990.

[7] R. Horaud and F. Veillon, "Finding geometric and relational structures in an image", Proc. of First ECCV, pp. 374-384, Antibes, France, 1990.

[8] F. Meyer and P. Maragos, "Morphological scalescale representation with levelings", Scale-space'99, LNCS, pp. 187-198, Spinger-Verlag, 1999.

[9] T.Y. Kong and A. Rosenfeld, "Digital topology: Introduction and survey", CVGIP, vol. 48, pp. 357-393, 1989.

[10] A. Manzanera, "Vision artificielle rétinienne", phD Thesis, ENST, 2000.

[11] A. Manzanera and T.M. Bernard, "Metrical properties of a family of 2D parallel thinning algorithm", IWCIA, Electronic Lecture Notes on Discrete Applied Mathematics, Elsevier Science, Palermo, May 2003.

[12] H.P. Moravec, "Visual mapping by a robot rover", Proc. of the 7th IJCAI, pp. 598-600, 1979.

[13] C. Schmid et al., "Comparing and evaluating interest points", ICCV, Bombay, January, 1998.

[14] J. Serra, Image analysis and mathematical morphology, New York, Academic Press, 1982.

[15] X. Zhang and D. Zhao, "A morphological algorithm for detecting dominant points on digital curves", SPIE Proc. Non Linear Image Processing VI, vol. 2424, pp. 372-383, 1995. 